\title{
The Problem With the Relativity of Simultaneity
}

\author{
Declan Traill ${ }^{1}$ \\ ${ }^{1}$ Independent Researcher, Melbourne, Australia \\ Correspondence: Declan Traill, Independent Researcher, Melbourne, Australia.
}

Received: January 25, 2022

Accepted: February 20, 2022

Online Published: March 2, 2022

doi:10.5539/apr.v14n1p26

URL: https://doi.org/10.5539/apr.v14n1p26

\begin{abstract}
Relativity claims that the simultaneity between two (or more) observers, each traveling in different Inertial Reference Frames (IRFs) is such that for two spaceships moving through space at a high-speed relative to one another "inside the frame of reference of Ship A, everything is moving normally, but everything over on Ship B appears to be moving slower (and vice versa)". However, as I will explain, this interpretation leads to an inconsistency which cannot be true. I point out the error being made in the interpretation of Minkowski diagrams that leads to this inconsistency, and how the diagram should be interpreted to correct this error. This paper demonstrates that a moving object's rate of time is determined based on its speed relative to a stationary reference frame and that the light signals propagating between objects (from which observers can determine the other object's rate of time) move at the speed of light c with respect to this stationary frame. If two objects are moving at the same speed through the stationary frame (but in different directions to each other) then they will have the same degree of time dilation and will thus have the same rate of time, despite the relative motion that exists between them.
\end{abstract}

Keywords: relativity, simultaneity, time, dilation, simultaneous, events, classical, physics, spaceships, clocks, Lorentz, transformations

\section{Introduction}

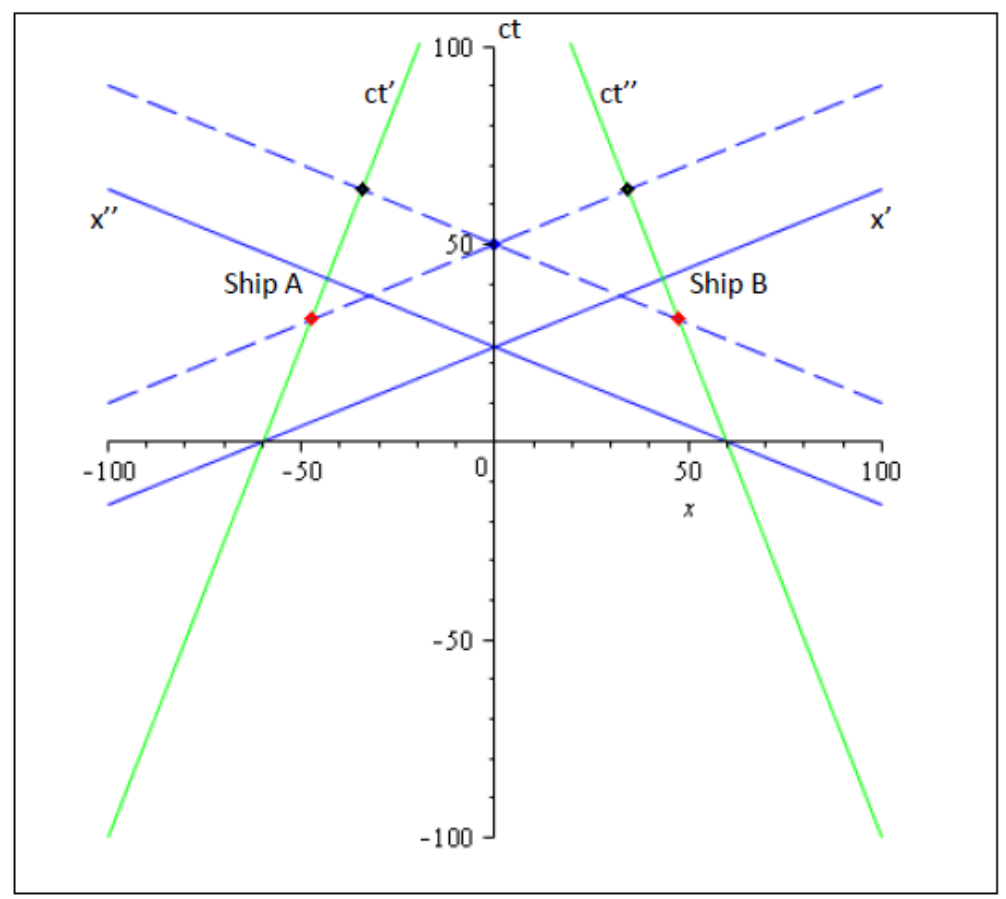

Figure 1 
This diagram (Figure 1) is a Minkowski diagram depicting two spaceships approaching each other at $40 \%$ of the speed of light. Ship A is moving from left to right and ship B from right to left. The Black axes are for a stationary observer between ships A and B (y axis is ct, $\mathrm{x}$ axis is distance). The Green axes are ship A \& B's ct' and ct" respectively and the blue axes are ship A \& B's x' and x" respectively. The dashed blue lines are parallel to x' and x" and are lines of simultaneity for each of the ships (A and B). These dashed lines pass through an event on the stationary observer's ct axis (at coordinates 0,50 ), where the event is indicated by a blue dot.

Minkowski diagrams are used to depict events occurring in space-time and can simultaneously represent different inertial reference frames. The orthogonal axes of the diagram represent the inertial reference frame of the observer. The Horizontal $\mathrm{X}$ axis represents spatial distance and the vertical $\mathrm{Y}$ axis represents time (often referred to as the 'ct' axis, as multiplying the time by speed of light $\mathrm{c}$ then converts a time into a similarly scaled distance which can be represented on the diagram). Other objects that are moving relative to that observer are represented by points somewhere on the diagram, corresponding to a certain space-time coordinate in the stationary observer's space. These points have their own axes representing distance and time for an observer on the moving object. However, due to the Lorentz Transformations (and Relativity), the axes are not geometrically orthogonal but are angled (by an equal amount) towards a line at 45 degrees from the horizontal and vertical axes of the stationary observer. These angled axes are not geometrically orthogonal, but due to the Lorentz transformations they are mathematically orthogonal for the moving inertial reference frame.

This 45-degree line represents something (light) moving at the speed of light, so the faster the object travels, the closer the axes get to being along the 45-degree line. For a moving observer, events in the stationary observer's reference frame that have the same time all lie along lines that are parallel to his own space $\mathrm{X}$ axis. These lines are known as lines of simultaneity as all events occurring along these lines are simultaneous from the point of view of the moving observer. Similarly, events that all occur at the same location in space all lie along lines that are parallel to his own ct axis.

As the axes of the different inertial reference frames in the diagram are not the same, determining the location and time of an event from the point of view of different observers in different inertial reference frames is not simply a matter of reading off the coordinates of the event on the orthogonal axes of the stationary observer. This will only give the space-time coordinate for the stationary observer's point of view. Different observers in different inertial reference frames will determine the space-time coordinates to be different due to their motion and their own angled space \& time axes.

These lines of simultaneity work fine for moving observers relative to the stationary reference frame, but as I will demonstrate, lines of simultaneity cannot be used to make such simple inferences about the simultaneity of events between reference frames that are both moving relative to the stationary reference frame (unless both moving observers have the same speed and direction as one another). For example, in the case being considered in this paper - that of two spaceships approaching each other, each traveling at $40 \%$ of the speed of light through the space of the stationary observer - the lines of simultaneity from one spaceship cannot be drawn into the space defined by the axes of the other spaceship and make meaningful (or correct) inferences about the time of the events in the other reference frame. The two sets of axes represent different coordinate systems and a mapping is required when changing from one to the other.

The original discussion about the Relativity of Simultaneity by Einstein using the example of a train passing by an embankment when two strikes of lightning occur (Einstein. A, 1948) serves to illustrate that events that are simultaneous in one reference frame need not be simultaneous in another reference frame. This much I agree with, and the use of Minkowski diagrams is correct where one observer is stationary and the other is moving [Yousuf. M. A, Woiciechowski., 2016]. There is a difference in perception between the stationary reference frame and a moving reference frame. However, this line of reasoning (with the use of Minkowski diagrams to draw conclusions) has been extended improperly into situations that, in my opinion, are incorrectly analysed, leading to incorrect conclusions about the rates of time between moving reference frames (Ashwinkumar. M, 2015).

Relativity asserts that where the lines of simultaneity of each ship (A or B) pass through the other ship's (B or A) ct axis (indicated by the Black dots in Fig. 1) indicates what time, on that second ship, is simultaneous with the first ships ct intersect. Therefore, so Relativity claims, ship A observes ship B (and vice-versa) to have a different time, and rate of time, than his own. In the words of this reference (Lumen Learning, 2021) "inside the frame of reference of Ship A, everything is moving normally, but everything over on Ship B appears to be moving slower (and vice versa)". However, it is easy to prove that this interpretation is wrong: If each of the 
three observers (ship A, ship B and the stationary observer in the middle) send signals to each other indicating their own current time, then each of the signals form ship A and ship B arrive at the event on the stationary observer's ct axis (indicated by a blue dot in Figure 1).

Simultaneously, the stationary observer's time signal travels from the blue dot to each of ship A's and B's ct axes (indicated by the Red dots in Figure 1). As all three of these points are connected by lines of simultaneity, all readings correspond to the same moment (although each observer's clock may show a different time). Each ship sends its current time to the observer at the Blue dot and he displays the times on a screen visible to each ship. Each ship sees the screen at a time simultaneous with the Red dot on his own ct axis. So, as the points indicated by the Red dots are simultaneous with the blue dot event on the stationary observer's ct axis, the points indicated by Black dots CANNOT also be simultaneous with these events as Relativity claims. Only one time on each of ct' and ct' axes can be simultaneous with an event on the stationary observer's ct axis.

So, to correctly determine the time that is simultaneous on ship A from ship B's point of view (and vice-versa), one must follow the dashed blue line of simultaneity from ship B up to the point where it intersects with ship A's line of simultaneity (the other dashed Blue line). For any situation involving two approaching or receding ships, one can then draw a stationary IRF (drawn on the diagram as orthogonal $\mathrm{x}$ and ct axes) at this point of intersection, representing a stationary observer between the two ships, in the way I have done in Figure 1. Then, to determine the time on ship A's ct' axis that is simultaneous with ship B's Red dot event (on his ct'” axis), one must follow ship A's line of simultaneity until it intersects with ship A's ct axis.

\section{Determining Signal Emission Times}

Analysing this situation with actual light signals, which take time to propagate, means that on reception of a signal by an observer, he must apply a correction to the arrival time in order to determine what the emission time (in his own reference frame) was. As the Minkowski diagram is drawn from the point of view of the stationary observer, he must take his own motion into consideration in order to apply the correction properly. First, he must determine the travel time of the light signal (as determined from the Minkowski diagram using the stationary observer's axes).

As the light signal travels at 45 degrees in any IRF on a Minkowski diagram, the observer on Ship B simply traces the light path back in time until it intersects the known trajectory of Ship A. This point must then indicate where and when the light signal was emitted. Then he must determine the distance on the Minkowski diagram (drawn from the stationary observer's perspective) to trace back along his ct axis. This can be done by multiplying the time interval $d t$ by the length along his ct axis corresponding to one unit of time on the stationary observer's ct axis $\left(h_{B}\right.$, the length of the hypotenuse of a triangle with side lengths of 1 and $\beta$ ).

To determine his actual time at the moment the light signal was emitted, he must divide the distance along his ct axis (just determined) by the unit time length on his ct axis (given by $\mathrm{U}$ ). For moving inertial reference frames, $\mathrm{U}$ will be greater than 1 . Once this is done, he must subtract this result from his measured light arrival time in order to know what his own time was when the light was emitted.

The time correction to determine Ship B's time is:

$$
\Delta t=-\frac{h_{B} \cdot d t}{U_{B}}=-\frac{d t}{\gamma}
$$

Where:

$d t$ is the travel time of the light signal (using the stationary observer's axes).

B subscript refers to values for the observer of the light signal.

$h_{B}$ refers to the length of the hypotenuse of a triangle with side lengths of 1 and $\beta$.

$$
\begin{gathered}
\beta=\frac{v}{c} \\
h_{B}=\sqrt{1+\beta^{2}} \\
\gamma=\frac{1}{\sqrt{1-\beta^{2}}} \\
U_{B}=h_{B} \cdot \gamma=\sqrt{\frac{1+\beta^{2}}{1-\beta^{2}}}
\end{gathered}
$$


Note: For source and destination reference frames that have different non-zero speeds, in order to map from one to the other and get the correct distance to move back along the observer's ct axis, one must account for the time shift from one IRF coordinate system (the origin of the signal) and another IRF coordinate system (the observer's frame). This difference is due to the difference in the angles of each frame's x axis (due to their different speeds), thus the different points at which each intersects with the stationary observer's ct axis. So, to map from one IRF to another on the Minkowski diagram, the dt value used in the above equations must have this mapping adjustment $t_{A B}$ added to it:

If

$$
\begin{gathered}
\beta_{A} \neq 0 \text { and } \quad \beta_{B} \neq 0 \\
t_{A B}=\left(\beta_{A} \cdot x_{A}-\beta_{B} \cdot x_{B}\right)
\end{gathered}
$$

Where: $\quad x$ is the coordinate along the $\mathrm{X}$ axis (positive or negative distance from the $\mathrm{Y}$ axis)

A refers to the sender of the signal

$\mathrm{B}$ refers to the observer of the signal

Thus, the full form of the time correction equation is:

$$
\Delta t=-\frac{h_{B} \cdot\left(d t+t_{A B}\right)}{U_{B}}=-\frac{d t+\left(\beta_{A} \cdot x_{A}-\beta_{B} \cdot x_{B}\right)}{\gamma}
$$

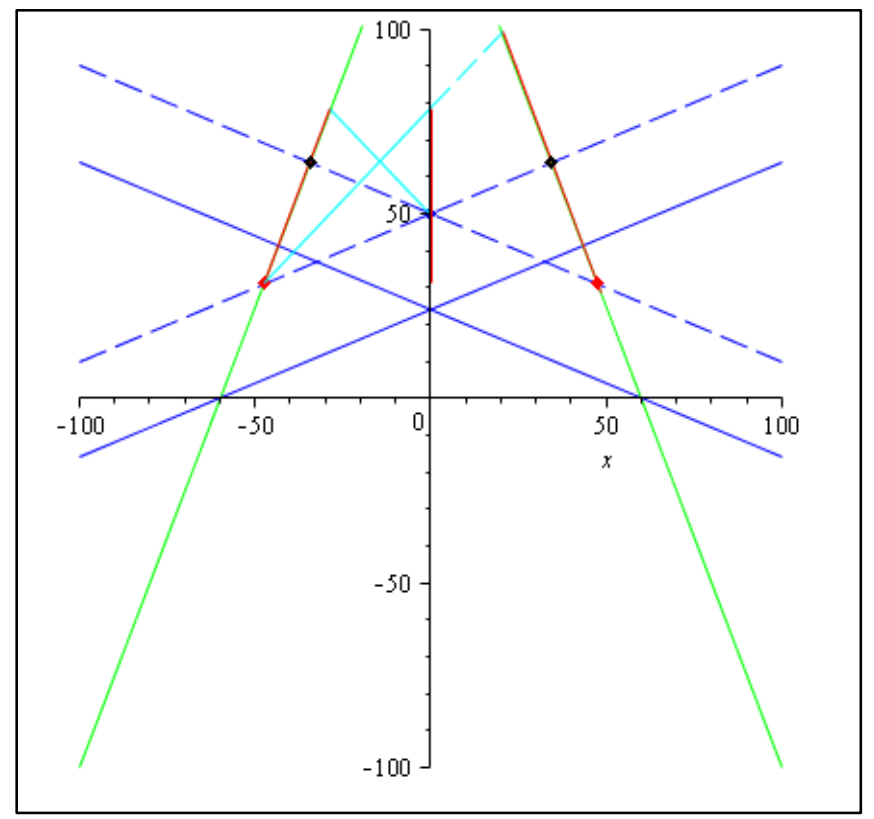

Figure 2

This diagram shows two light signals (in Cyan). One from Ship A to the stationary observer at coordinate $(0,50)$, and another from the stationary observer to Ship A. Also shown in Red is the correction made by each observer to the time of the received light signal, such that he can determine when the signal was sent. Also shown is the light signal from Ship A continuing to reach Ship B after passing the stationary observer (dashed cyan line). Then on Ship B's ct axis he applies his time correction for the entire travel time of the light signal (the time to go from Ship A to the stationary observer, plus the time to go on from there to Ship B's ct axis). As you can see, the observer on Ship B determines that the time on Ship A is the same as his own. 


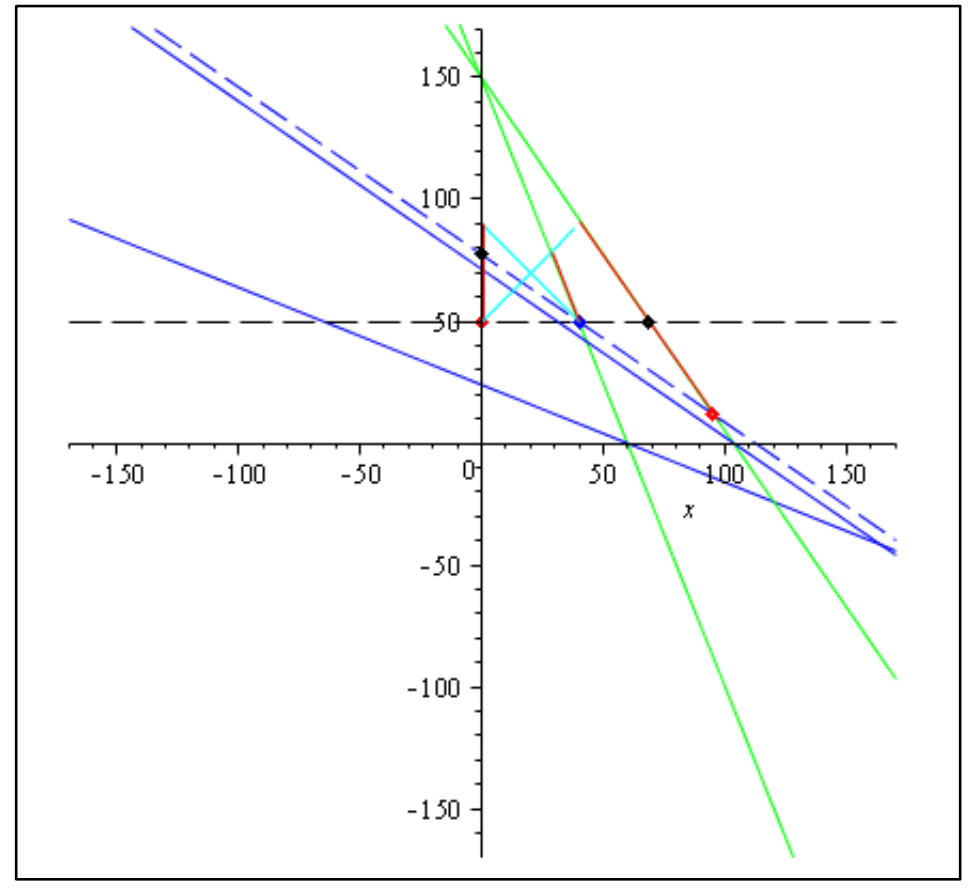

Figure 3

This diagram shows the same information as in Figure 2 but re-drawn from the point of view of Ship A (Ship A's Inertial Reference Frame).

\section{Discussion}

Furthermore, if the claim is made that the different time observed from one ship to the other are just what is OBSERVED rather than real time differences, then that too is incorrect as the lines of simultaneity depict actual simultaneity of events and there is no propagation delay due to the travel time of light included in the diagram. For the difference in time to be just an observational difference one would have to explain the difference between observed time and actual time to be due to the time taken for the light signal to travel from one ship to the other, but I have just shown that even if real light signals are used, both ships determine that their times, and rates of time are the same.

The rate of time of each IRF (relative to the observer's IRF) can be determined, even without clock synchronization, by comparison of two or more readings of the other IRF's times over a known local time interval in the observer's IRF. If this is done, then ship A's observer will see the rate of time on ship B to be the same as his own.

So, if ship A and B can determine that they have the same rate of time when they move towards or away from each other at the same speed through the space/medium field, then if they move together until they are both at a point on the Black ct axis, they can then compare actual clock times. Their respective times on their clocks at that point provides each with a reference point to which they can calibrate all the time observations they have previously made, and continue to make, on their journey towards and away from each other. After synchronizing their clocks to the same time, they then continue past each other whilst observing each other's time continually. They will then deduce that the two red dots on ct' and ct'" are simultaneous, not one Red dot and one Black dot as Relativity claims.

As a final clincher to the argument, as Relativity is founded on the Lorentz Transformations, they are the final arbiter as to what changes to time and length occur between moving Inertial Reference Frames. The Lorentz Transformations are equations that state how the time and length measures of one frame change when transformed to another frame. So, to provide a definitive answer as to what the rate of time of Ship A is from Ship B's perspective (and vice versa) one must perform the Lorentz Transformations from one frame to the other. I have done precisely this calculation and present the full calculation of this in Appendix A. From this calculation it is unequivocal that the rate of time of Ship A is the same as it is on Ship B and that each Ship is an 
equal distance from the $\mathrm{Y}$ axis of the stationary observer in the middle. It is also clear that both Ship A and Ship B's times are dilated (running slow) by the same amount, when compared to the clock of the stationary observer in the middle. This evidence should be indisputable, even by Relativists given that Relativity is founded on the Lorentz Transformations!

\section{Conclusion}

This misunderstanding about the Relativity of simultaneity stems from Einstein's mistaken assumption that the speed of light is really constant in any IRF and moves through space at speed c with respect to that IRF, rather than just measured to be so (as I have shown in previous work (Traill. D. A, 2012)) and actually has a fixed speed of $\mathrm{c}$ with respect to the space/medium field. The problem in the interpretation of Minkowski diagrams is due to the failure to recognize that each ship's axes represent a different coordinate system and one must map from one coordinate system to the other coordinate system when drawing inferences between the two systems. The Lorentz Transformation equations, upon which Relativity theory is built, have been used to demonstrate that the assertion made by Relativity regarding the rate of clocks on moving bodies (spaceships in this example) is incorrect and that the correct explanation for the rates of time on the moving bodies is only obtained when one realizes that there is a preferred inertial reference frame and that every body has a state of motion with reference to that preferred frame. This paper outlines a clear case for the theory in this type of situation. It would be interesting and useful if some experimental evidence could be measured to confirm these findings. There is already experimental evidence for the existence of a preferred inertial reference frame relative to which light's speed is set at c (Cahill R. T, 2009) and it is possible to reconstruct Relativity theory and explain its kinematic effects from the perspective of a Classical field theory with a preferred inertial reference frame due to the Gravitational potential field in a way similar to Mach's principle (Traill. D. A, 2012).

\section{References}

Ashwinkumar, M. (2015). Relativistic spaceships moving towards each other. Physics Stack-Exchange. Retrieved from https://physics.stackexchange.com/questions/209170/relativistic-spaceships-moving-towards-each-other/62 $0547 \# 620547$

Cahill, R. T. (2009). Combining NASA/JPL One-Way Optical-Fiber Light- Speed Data with Spacecraft Earth-Flyby Doppler-Shift Data to Characterise 3-Space Flow. Progress in Physics, 4. Retrieved 30 October, 2021 ,

from https://www.researchgate.net/publication/26842358_Combining_NASAJPL_One-Way_Optical-Fiber_Ligh t-Speed_Data_with_Spacecraft_Earth-Flyby_Doppler-Shift_Data_to_Characterise_3-Space_Flow

Einstein, A, (1948). Relativity: The Special and the General Theory, 17.

Lumen Learning. (2021). Boundless Physics. Consequences of Special Relativity. Retrieved 23 June 2021, from https://courses.lumenlearning.com/boundless-physics/chapter/consequences-of-special-relativity/

Traill, D. A. (2012). A Classical Reconstruction of Relativity. Retrieved 21 June 2021, from https://www.researchgate.net/publication/343975995_A_Classical_Reconstruction_of_Relativity

Yousuf, M. A., \& Woiciechowski, I. (2016). Spacetime Diagrams and Einstein's Theory For Dummies. Instructor's Solution Manual. Johns Hopkins University Center for Talented Youth, 11-13. Retrieved from https://www.google.com/url?sa=t\&rct=j\&q=\&esrc=s\&source=web\&cd=\&ved=2ahUKEwjx2vfAwIryAhW ryzgGHf26ARUQFnoECAUQAw\&url=http\%3A\%2F\%2Fpages.jh.edu\%2F maliyou $\% 2$ FLessons $\% 2$ FIns tructor $\% 2527 \mathrm{~s} \% 2520$ Solution $\% 2520 \mathrm{Manual} \% 2520$ for $\% 2520$ Space-time $\% 2520$ diagrams $\% 2520-\% 2520 \mathrm{Ver}$ \%25202.pdf\&usg=AOvVaw06UjWi-rdSreL5B37HiiZW 


\section{Appendix A}

\section{Proof That Ship A \& B Times Are at the Same Rate Using the Lorentz Transformations}

That Ship A and B have the same rate of time is easily proved using the Lorentz Transformations:

Transforming each ship to the IRF of the central observer, then eliminating the central IRF by substituting one Lorentz Transformation into the other:

\section{Note:}

For Ship A (single primed'): $\mathrm{v}$ is positive, $\Delta \mathrm{x}$ is negative

For Ship B (double primed"): $\mathrm{v}$ is negative, $\Delta \mathrm{x}$ is positive

For Time:

$$
\begin{aligned}
& \Delta \mathrm{t}^{\prime}=\gamma\left[\Delta \mathrm{t}-\mathrm{v} / \mathrm{c}^{2}(-\Delta \mathrm{x})\right] \\
& \Delta \mathrm{t}^{\prime \prime}=\gamma\left[\Delta \mathrm{t}-(-\mathrm{v}) / \mathrm{c}^{2} \Delta \mathrm{x}\right] \\
& \Delta \mathrm{t}=\Delta \mathrm{t}^{\prime \prime} / \gamma-\mathrm{v} / \mathrm{c}^{2} \Delta \mathrm{x} \\
& \Delta \mathrm{t}^{\prime}=\gamma\left[\left(\Delta \mathrm{t}^{\prime \prime} / \gamma-\mathrm{v} / \mathrm{c}^{2} \Delta \mathrm{x}\right)+\mathrm{v} / \mathrm{c}^{2} \Delta \mathrm{x}\right] \\
& \Delta \mathrm{t}^{\prime}=\gamma\left[\Delta \mathrm{t}^{\prime \prime} / \gamma\right] \\
& \Delta \mathrm{t}^{\prime}=\Delta \mathrm{t}^{\prime \prime}
\end{aligned}
$$

Thus, the rate of time for Ship A is the same as for Ship B.

For Space:

$$
\begin{aligned}
& \Delta \mathrm{x}^{\prime}=\gamma(-\Delta \mathrm{x}-\mathrm{v} \Delta \mathrm{t}) \\
& \Delta \mathrm{x}^{\prime \prime}=\gamma(\Delta \mathrm{x}-(-\mathrm{v}) \Delta \mathrm{t}) \\
& \Delta \mathrm{x}=\Delta \mathrm{x}^{\prime \prime} / \gamma-\mathrm{v} \Delta \mathrm{t} \\
& \Delta \mathrm{x}^{\prime}=\gamma\left(-\left(\Delta \mathrm{x}^{\prime \prime} / \gamma-\mathrm{v} \Delta \mathrm{t}\right)-\mathrm{v} \Delta \mathrm{t}\right) \\
& \Delta \mathrm{x}^{\prime}=-\gamma\left(\Delta \mathrm{x}^{\prime \prime} / \gamma\right) \\
& \Delta \mathrm{x}^{\prime}=-\Delta \mathrm{x}^{\prime \prime}
\end{aligned}
$$

This we know to be true, as each ship is at a point equidistant on either side of the origin.

\section{Copyrights}

Copyright for this article is retained by the author(s), with first publication rights granted to the journal.

This is an open-access article distributed under the terms and conditions of the Creative Commons Attribution license (http://creativecommons.org/licenses/by/4.0/). 